\title{
The active site residue tyrosine 325 influences iron binding and coupling efficiency in human phenylalanine hydroxylase
}

\author{
Frederico F. Miranda a , Matthias Kolberg b, K. Kristoffer Andersson ${ }^{\text {b }}$, \\ Carlos F.G.C. Geraldes ${ }^{\mathrm{c}}$, Aurora Martínez ${ }^{\mathrm{a}, *}$ \\ a Department of Biomedicine, University of Bergen, Jonas Lies vei 91, NO-5009 Bergen, Norway \\ ${ }^{\mathrm{b}}$ Department of Molecular Biosciences, University of Oslo, PO Box 1041 Blindern, NO-0316 Oslo, Norway \\ ${ }^{\mathrm{c}}$ Department of Biochemistry, NMR Center and Center of Neurosciences and Cell Biology, University of Coimbra, Portugal
}

Received 19 December 2004; received in revised form 21 February 2005; accepted 7 March 2005

Available online 28 April 2005

\begin{abstract}
Phenylalanine hydroxylase $(\mathrm{PAH})$ is a tetrahydrobiopterin $\left(\mathrm{BH}_{4}\right)$-dependent enzyme that catalyzes the hydroxylation of $\mathrm{L}-\mathrm{Phe}$ to L-Tyr. The non-heme iron in the enzyme (Fe(III) as isolated) is 6-coordinated to a 2-His-1-carboxylate motif and three water molecules (wat1, wat2 and wat3). Tyr325 is at the second coordination sphere, hydrogen-bonded to water (wat1). We prepared and expressed mutants with Leu, Ala, Ser and Phe at this position. Only Y325L and the conservative mutation Y325F resulted in stable enzymes, but the mutant Y325F has been found to be post-translationally hydroxylated and to revert back to wild-type PAH [S.D. Kinzie, M. Thevis, K. Ngo, J. Whitelegge, J.A. Loo, M.M. Abu-Omar, J. Am. Chem. Soc. 125 (2003) 4710-4711], being inadequate to investigate the early inferred functional role of Tyr325. On the other hand, compared to wild-type PAH, Y325L shows reduced specific activity, decreased coupling efficiency and decreased iron content. The mutant also reveals a very high affinity for L-Phe and $\mathrm{BH}_{4}$ and does not manifest positive cooperativity for the substrate. All together, our results support that the mutation Y325L causes the removal or increased delocalization of the iron-ligated wat 1 and, in turn, a less tight binding of the metal. Tyr325 thus appears to have an important role ensuring stoichiometric binding of iron, correct geometry of the complexes with substrate and cofactor and, consequently, a right coupling efficiency of the PAH reaction. In addition, the residue appears to be important for the correct cooperative regulation by L-Phe.
\end{abstract}

(C) 2005 Elsevier Inc. All rights reserved.

Keywords: Phenylalanine hydroxylase; Non-heme iron; Coupling efficiency; Coordination sphere, EPR; Tetrahydrobiopterin

\section{Introduction}

Phenylalanine hydroxylase (PAH, EC 1.14.16.1) catalyzes the hydroxylation of L-Phe to L-Tyr. This reaction is the rate-limiting step in the catabolism of L-Phe, and mutations in the human PAH gene result in phenylketonuria, which is the most common inherited disease of amino acid metabolism [1]. The human enzyme is tetrameric and contains an essential non-heme iron per

\footnotetext{
${ }^{*}$ Corresponding author. Tel.: +47 55586427; fax: +4755586360.

E-mail address: aurora.martinez@biomed.uib.no (A. Martínez).
}

subunit. The reaction requires molecular oxygen and a pterin cofactor which in fact functions as a cosubstrate [2].The natural cofactor of PAH is tetrahydrobiopterin $\left(\mathrm{BH}_{4}\right)$, and during hydroxylation of L-Phe, the dioxygen is partitioned between the phenolic hydroxyl group of tyrosine and the 4a-hydroxyl group of 4a-carbinolamine (4-HO- $\left.\mathrm{BH}_{4}\right)$, which is then recycled back to $\mathrm{BH}_{4}$ via 4a-carbinolamine dehydratase and dihydropteridine reductase (DHPR) [2]. PAH is regulated by its substrate L-Phe, which activates the enzyme and elicits positive cooperativity; $\mathrm{BH}_{4}$ serves as a negative effector in addition to reductively activate $\mathrm{PAH}[2,3]$. 
From the crystal structure of the catalytic domain of human $\mathrm{PAH}$, it is seen that the iron is coordinated to Glu330, His285, His290 and three water molecules, wat1, wat2 and wat3 [4] (Fig. 1(a)). Tyr325 is conserved in all mammalian PAH and is located at the second coordination sphere of the iron, hydrogen bonded to wat 1 . The apparently central structural role of Tyr325 as well as its proximity to the iron early induced the suggestion that this residue had an important catalytic role in the PAH reaction [4]. Furthermore, the structures of the binary complexes of $\mathrm{PAH}$ with either $\mathrm{BH}_{4}[5]$ or the oxidized cofactor analogue dihydrobiopterin $\left(\mathrm{BH}_{2}\right)[6]$ showed the carbonyl at $\mathrm{C} 4$ of the pterin hydrogen bonding to wat1. This hydrogen bonding network involving Tyr325 thus appears to have a role in stabilizing the active site and positioning the pterin cofactor. Extensive mutagenesis studies at Tyr325 in the catalytic domains of either human $\mathrm{PAH}$ [6] or rat $\mathrm{PAH}$ [7], or at the corresponding Tyr371 in the full length rat homologous enzyme tyrosine hydroxylase [8], did not unambiguously confirm the significance of this residue in catalysis or

(a)

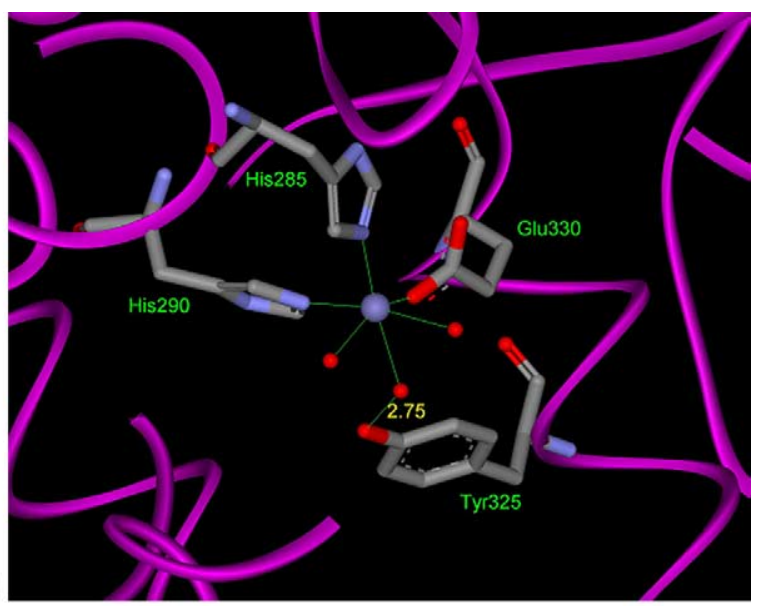

(b)

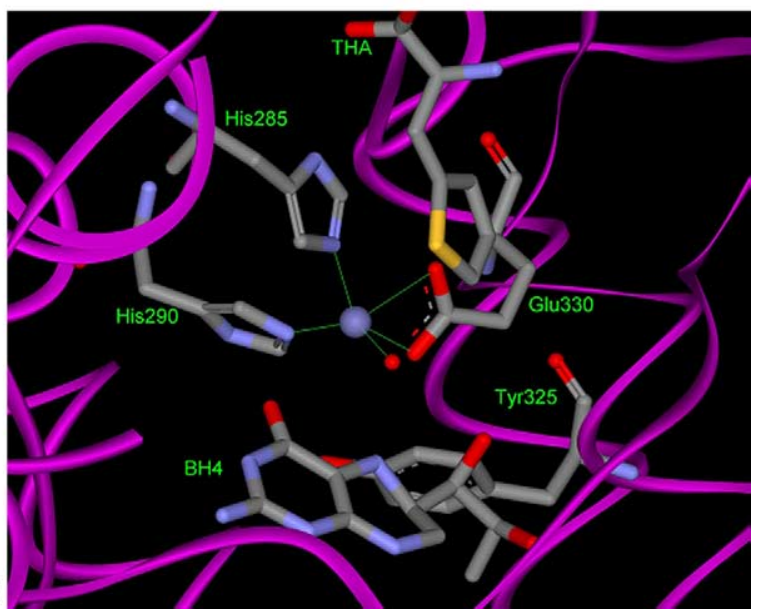

Fig. 1. Close up on PAH active site structure in the absence (a) (PDB $1 \mathrm{PAH})$ and in the presence of the L-Phe analogue 3-(2-thienyl)-Lalanine and $\mathrm{BH}_{4}$ (b) (PDB $\left.1 \mathrm{KW}\right)$ ). The iron is shown with its first coordination sphere. Tyr325 is also shown. in the binding of Phe and $\mathrm{BH}_{4}$. Notably, the conservative mutation Tyr to Phe resulted in enzymes of similar activity and affinity for the natural substrates. This intriguing result was explained by Kinzie et al. showing that the Y325F mutant in human PAH was rescued by self-hydroxylation [9]. Posttranslational self-hydroxylation reactions occur in several non-heme iron enzymes (for review see [10]). The posttranslational oxidative repair of the Y325F PAH mutant to retain wild-type structure again indicates the functional importance of Tyr325. Accordingly, the mutation of Tyr to Phe in the corresponding residue (Tyr179) in Chromobacterium violaceum $\mathrm{PAH}$, which is not the subject of self hydroxylation, significantly decreases the catalytic activity while increasing the apparent affinity for L-Phe [11]. The same catalytic effects accompanied the mutation of $\mathrm{Y} 325 \mathrm{~L}$ in rat PAH [7].

We aimed to further investigate and clarify the structural and catalytic role of Tyr325 in human PAH and prepared several mutants at this position, in addition to $\mathrm{Y} 325 \mathrm{~F}$, in the full-length form of the enzyme. The kinetic properties of the mutants have been studied in detail, and electron paramagnetic resonance (EPR) spectroscopy has been applied to characterize iron binding and iron coordination geometry. The stable mutant Y325L turned out to be very valuable in this respect and allowed us to characterize the role of this residue at the second coordination sphere on the iron binding stoichiometry, the positive regulation by the substrate L-Phe and the coupling efficiency of the reaction.

\section{Experimental}

\subsection{Site-directed mutagenesis}

The mutations Y325A, Y325F, Y325L and Y325S were introduced in the human PAH cDNA by polymerase chain reaction (PCR)-based site-directed mutagenesis using the QuikChangeTM kit (Stratagene). The primers used for mutagenesis (provided by MWG Biotech AG, Ebersberg) are shown in Table 1. The authenticity of the mutagenesis was verified by DNA sequencing using the primers described previously [12].

\subsection{Expression and purification of the enzymes}

Transformed Escherichia coli with the pMAL vectors were grown and the enzymes were purified by affinity chromatography on amylose resin (New England Biolabs) as described [13]. After cleaving the fusion protein with enterokinase (EKMax ${ }^{\mathrm{TM}}$ from Invitrogen) using 20 units $\mathrm{EKMax}^{\mathrm{TM}} / \mathrm{mg}$ of fusion protein at $4{ }^{\circ} \mathrm{C}$ for $4 \mathrm{~h}$, the tetrameric enzyme forms were isolated by size exclusion chromatography as described [13]. 
Table 1

Primers used for the PCR site-directed mutagenesis

\begin{tabular}{|c|c|c|}
\hline Mutant & Sense & Sequence \\
\hline Y325A & Forward & 5'-AAG-CTC-GCC-ACA-ATT-GCC-TGG-TTT-ACT-GTG-3' \\
\hline Y325A & Reverse & 5'-CAC-AGT-AAA-CCA-GGC-AAT-TGT-GGC-GAG-CTT-3' \\
\hline Y325F & Forward & 5'-AAG-CTC-GCC-ACA-ATT-TTC-TGG-TTT-ACT-GTG-3' \\
\hline $\mathrm{Y} 325 \mathrm{~F}$ & Reverse & 5'-CAC-AGT-AAA-CCA-GAA-AAT-TGT-GGC-GAG-CTT-3' \\
\hline Y325L & Forward & 5'-AAG-CTC-GCC-ACA-ATT-CTC-TGG-TTT-ACT-GTG-3' \\
\hline Y325L & Reverse & 5'-CAC-AGT-AAA-CCA-GAG-AAT-TGT-GGC-GAG-CTT-3' \\
\hline Y325S & Forward & 5'-AAG-CTC-GCC-ACA-ATT-TCC-TGG-TTT-ACT-GTG-3' \\
\hline Y325S & Reverse & 5'-CAC-AGT-AAA-CCA-GGA-AAT-TGT-GGC-GAG-CTT-3' \\
\hline
\end{tabular}

The codon changes are shown in bold type.

\subsection{Assay of PAH activity}

PAH activity was assayed at $25^{\circ} \mathrm{C}$ with a standard reaction mixture (final volume, $50 \mu \mathrm{l}$ ) of $10 \mu \mathrm{M}$ ferrous ammonium sulfate, $1 \mathrm{mM}$ L-Phe, $5 \mathrm{mM}$ dithiothreitol, and $75 \mu \mathrm{M} \mathrm{BH}_{4}(6(R)$-L-erythro-5,6,7,8-tetrahydrobiopterin from Schirck's, Laboratories, Jona, Switzerland), $0.04 \mu \mathrm{g} / \mu \mathrm{l}$ catalase, $0.05 \%(\mathrm{w} / \mathrm{v})$ bovine serum albumin, and $1 \mu \mathrm{g}$ of the various human PAH forms in $100 \mathrm{mM}$ Na- $N$-2-hydroxyethylpiperazine- $N N^{\prime}$-ethanesulphonic acid (HEPES), pH 7.0, as described [13,14]. The kinetic parameters were determined using variable concentrations of the substrate $(0-5 \mathrm{mM}$ L-Phe with $75 \mu \mathrm{M}$ $\left.\mathrm{BH}_{4}\right)$ and of the cofactor $(0-500 \mu \mathrm{M} \mathrm{BH} 4$ with $1 \mathrm{mM}$ L-Phe) and were calculated by non-linear regression analysis of the experimental data using the program SigmaPlot 2000 (SPSS Inc.).

\subsection{PAH/Dihydropteridin reductase coupled assay}

A coupled assay with DHPR was used to determine the ratio of L-Phe conversion to L-Tyr to the oxidation of $\mathrm{BH}_{4}$ (in $\mu \mathrm{mol} / \mu \mathrm{mol}$, i.e., coupling efficiency) [15]. The assay mixture consisted of $0.01 \mathrm{mg} / \mathrm{ml} \mathrm{PAH,}$ $100 \mu \mathrm{M}$ ferrous ammonium sulfate, $1 \mathrm{mM}$ Phe, $100 \mu \mathrm{M}$ $\mathrm{BH}_{4}, 1.24$ U DHPR (Sigma), $200 \mu \mathrm{M}$ NADH (Sigma), $0.1 \%(\mathrm{w} / \mathrm{v})$ bovine serum albumin and $0.04 \mu \mathrm{g} / \mu \mathrm{l}$ catalase in $250 \mathrm{mM}$ Na-HEPES, pH 7.0. The enzymes were preincubated with $1 \mathrm{mM}$ L-Phe $\left(5 \mathrm{~min}, 25^{\circ} \mathrm{C}\right)$. Oxidation of $\mathrm{BH}_{4}$ was determined by monitoring the decrease in absorbance at $340 \mathrm{~nm}$ due to NADH oxidation $\left(\varepsilon=6220 \mathrm{M}^{-1} \mathrm{~cm}^{-1}\right)$ for $1 \mathrm{~min}$ at $25^{\circ} \mathrm{C}$ and the amount of L-Tyr formed at the end of the assay was measured by high liquid pressure chromatography and fluorimetric detection [13]. Background levels of oxidized pterin arising from autooxidation of reduced pterin were controlled for when calculating the levels of $\mathrm{BH}_{4}$ oxidation.

\subsection{Fluorescence measurements}

Measurements of intrinsic tryptophan fluorescence were performed at $25^{\circ} \mathrm{C}$ in $20 \mathrm{mM}$ Na-HEPES, $0.2 \mathrm{M}$
$\mathrm{NaCl}$ at $\mathrm{pH} 7.0$, with $1 \mu \mathrm{M}$ PAH subunit. A Perkin-Elmer LS-50 luminescence spectrometer with a constanttemperature cell holder and $1-\mathrm{cm}$ path-length quartz cells was used. The excitation and emission slits were 3 and 5, respectively. All spectra were corrected for blank emission.

\subsection{EPR spectroscopy and redox titrations}

Enzyme samples (200 $\mu \mathrm{M}$ subunit) for EPR analyses were prepared in $20 \mathrm{mM}$ Na-HEPES, $0.2 \mathrm{M} \mathrm{NaCl}, \mathrm{pH}$ 7.0. Additions and incubations ( $5 \mathrm{~min}, \mathrm{pH} 7.0,25^{\circ} \mathrm{C}$ ) with $5 \mathrm{mM}$ L-Phe and $5 \mathrm{mM}$ L-erythro-7,8-dihydrobiopterin $\left(\mathrm{BH}_{2}\right)$ were performed prior to freezing the samples. EPR analysis were performed at $9.66 \mathrm{GHz}$ microwave frequency on a Bruker ESP300E equipped with an Oxford Instruments helium flow cryostat ESR 900 at $3.6 \mathrm{~K}$ and a Bruker dual mode resonator ER4116DM operated at the perpendicular mode $\left(\mathrm{TE}_{102}\right)$. Other EPR parameters are given in the figure legends. The EPR spectra were smoothed with the polynomial filter $(n=5-15)$ provided in the WINEPR software (Bruker) and were base-line corrected.

Potentiometric titrations for subsequent EPR monitoring were performed in a 2-ml anaerobic cell under purified argon at $4{ }^{\circ} \mathrm{C}$. The bulk potential of the stirred solution was measured using a platinum wire electrode with respect to the potential of a Red Rod microsample electrode REF200 from Radiometer Analytical. Reported potentials were all expressed relative to the normal hydrogen electrode. PAH samples $(100 \mu \mathrm{M}$ subunit) were poised at various potentials in the presence of $100 \mu \mathrm{M}$ each of the following mediators: $N, N, N^{\prime}, N^{\prime}$-tetramethyl-p-phenylenediamine, 2,6-dichlorophenolindophenol, phenazine ethosulfate, methylene blue, resorufin, indigosulfonate, phenosafranin, safranin $\mathrm{O}$, neutral red, benzyl viologen, and methyl viologen [16]. Potassium ferricyanide (III) and sodium dithionite were used as oxidant and reductant, respectively. Redox equilibrium was obtained as judged by the attainment of a stable solution potential within a few minutes after the addition of the titrant to the reaction mixture. The samples were transferred anaerobically to EPR tubes and 
directly frozen in liquid nitrogen. Midpoint potentials $\left(E_{\mathrm{m}}\right)$ were obtained from the experimental data points with a least squares fit to the Nernst equation $(n=1)$. The EPR monitored titrations of wt-PAH and mutant samples were performed at a microwave frequency of $9.66 \mathrm{GHz}$ and a microwave power of $1 \mathrm{~mW}$, with a $10-$ $\mathrm{G}(0.1 \mathrm{mT})$ modulation amplitude and a modulation frequency of $100 \mathrm{kHz}$ at $3.6 \mathrm{~K}$. $P_{1 / 2}$ was determined by least square fitting (by using Origin version 6.1, OriginLab Corporation) to the saturation curve described by Eq. (1), where $I$ is the EPR amplitude, $P$ is the microwave power and the parameter $b$ describes the contribution of inhomogeneous broadening [17]:

$I \propto 1 /\left(1+P / P_{1 / 2}\right)^{\mathrm{b} / 2}$.

\subsection{Iron quantification}

Iron quantification by chelation with bathophenanthrolinedisulphonic acid was performed essentially as described [18]. $15 \mu \mathrm{M}$ subunit of wt-PAH and mutants were incubated with $1 \mathrm{mM}$ L-Phe, $60 \mu \mathrm{M} \mathrm{BH}, 1 \mathrm{mM}$ dithiothreitol at $25^{\circ} \mathrm{C}$, and, after $3 \mathrm{~min}, 50 \mathrm{mM}$ bathophenanthrolinedisulphonic acid (Sigma) was added to form the $\mathrm{Fe}(\mathrm{II})$-bathophenanthroline complex and determine the iron content using a $\varepsilon_{535}=$ $22,000 \mathrm{M}^{-1} \mathrm{~s}^{-1}$ [19]. Quantification of the EPR visible $\mathrm{Fe}(\mathrm{III})$ was performed as described [16], applying the method developed by Aasa and Vänngård $[20,21]$ to regions of EPR spectra taken at several temperatures between 3.6 and $25 \mathrm{~K}$, and using transferrin and ferric myoglobin $(100-200 \mu \mathrm{M})$ as references for the $g=4.3$ and $g=6.0$ iron species, respectively.

\section{Results}

\subsection{Expression and purification}

The full-length PAH mutants Y325A, Y325F, Y325L and $\mathrm{Y} 325 \mathrm{~S}$ were obtained by site-directed mutagenesis. All these enzyme forms expressed well in $E$. coli, but
Y325S quickly aggregated after purification and was not suitable for a detailed characterization. Y325A also showed a high degree of aggregation but we were able to obtain sufficient tetrameric form for a partial characterization of the steady-state kinetic properties. The mutants $\mathrm{Y} 325 \mathrm{~F}$ and $\mathrm{Y} 325 \mathrm{~L}$ were stable and were obtained at similar yields and oligomeric distribution as wild-type (wt) PAH, consisting mainly of tetrameric forms which were purified to homogeneity.

\subsection{Steady-state kinetic analysis of the hydroxylating reaction}

In order to exhibit maximal specific activity with the natural cofactor $\mathrm{BH}_{4}, \mathrm{PAH}$ must be preincubated with its substrate L-Phe, which binds with positive cooperativity (Hill coefficient, $h \approx 2$ ) [3] and induces a regulatory activating conformational change and 3-fold increase in the activity. The specific activities for the enzyme forms prior to and after preincubation with L-Phe are summarized in Table 2. As expected [9], Y325F showed indistinguishable properties to wt$\mathrm{PAH}$. On the other hand, the mutant Y325L appears to be appropriate to investigate the early suspected role of Tyr325 on the structure and function of the enzyme [4]. The mutation to Leu does not affect the conformational stability of the enzyme (see above) but the mutant shows lower hydroxylating activity and no cooperativity for L-Phe $(h=1.1)$ (Fig. 2 and Table 2$)$. Remarkably, this mutant displays a significantly lower $S_{0.5}(\mathrm{Phe}$ )-value (concentration of L-Phe giving half-maximal activity), approaching the value for the activated forms [22]. This increase in affinity is accompanied by a concomitant increase in substrate inhibition, which is manifested at [L-Phe] $>500 \mu \mathrm{M}$, vs $1 \mathrm{mM}$ for wt-PAH (Fig. 2). The $K_{\mathrm{m}}\left(\mathrm{BH}_{4}\right)$ was also reduced in Y325L, indicating a higher affinity for both the substrate and cofactor (Table 2). The mutation of the tyrosine to alanine significantly reduced the activity of the enzyme and, as Y325L, Y325A did not show any further activation by preincubation with L-Phe (Table 2).

Table 2

Steady-state kinetic parameters for the tetrameric forms of wt-PAH and the mutants at position Tyr325

\begin{tabular}{|c|c|c|c|c|c|}
\hline \multirow[t]{2}{*}{$\overline{\text { Enzyme }}$} & \multicolumn{2}{|c|}{$V_{\max }^{\mathrm{a}, \mathrm{b}}(\mu \mathrm{mol} \mathrm{Tyr} / \mathrm{min} \mathrm{mg})$} & \multirow[t]{2}{*}{$S_{0.5}(\mathrm{~L}-\mathrm{Phe})(\mu \mathrm{M})$} & \multirow[t]{2}{*}{$K_{\mathrm{m}}\left(\mathrm{BH}_{4}\right)(\mu \mathrm{M})$} & \multirow[t]{2}{*}{$\overline{h \text { (L-Phe) }}$} \\
\hline & Non L-Phe activated $^{\mathrm{a}}$ & L-Phe activated ${ }^{\mathrm{b}}$ & & & \\
\hline $\mathrm{Wt}$ & $1230 \pm 300$ & $3640 \pm 360$ & $154 \pm 20$ & $28 \pm 7$ & 2.0 \\
\hline Y325F & $1150 \pm 220$ & $3630 \pm 150$ & $184 \pm 83$ & $27 \pm 2$ & 1.9 \\
\hline Y325L & $1310 \pm 130$ & $1500 \pm 90$ & $57 \pm 28$ & $8 \pm 1$ & 1.1 \\
\hline Y325A & $290 \pm 2$ & $300 \pm 24$ & $-{ }^{c}$ & $-^{c}+1$ & $-{ }^{c}$ \\
\hline
\end{tabular}

All measurements made at $25^{\circ} \mathrm{C}, \mathrm{pH}$ 7.0. $V_{\max }$ for non-L-Phe activated and $K_{\mathrm{m}}\left(\mathrm{BH}_{4}\right)$ were calculated without preincubation with L-Phe, at $1 \mathrm{mM}$ LPhe and variable concentrations of $\mathrm{BH}_{4}(0-200 \mu \mathrm{M}) . V_{\max }$ for L-Phe activated, ${ }^{\mathrm{b}}$ the concentration of L-Phe at half maximal activity $\left(S_{0.5}\right)$, and the Hill coefficient $(h)$ for L-Phe were determined at $75 \mu \mathrm{M} \mathrm{BH}_{4}$ and variable concentrations of L-Phe $(0-5 \mathrm{mM})$. The enzymes were preincubated for 5 min $\left(25^{\circ} \mathrm{C}, \mathrm{pH} 7.0\right)$ with L-Phe at the same concentrations as in the assay. ${ }^{\mathrm{c}}$ These parameters could not be determined for this mutant due to low activity. 


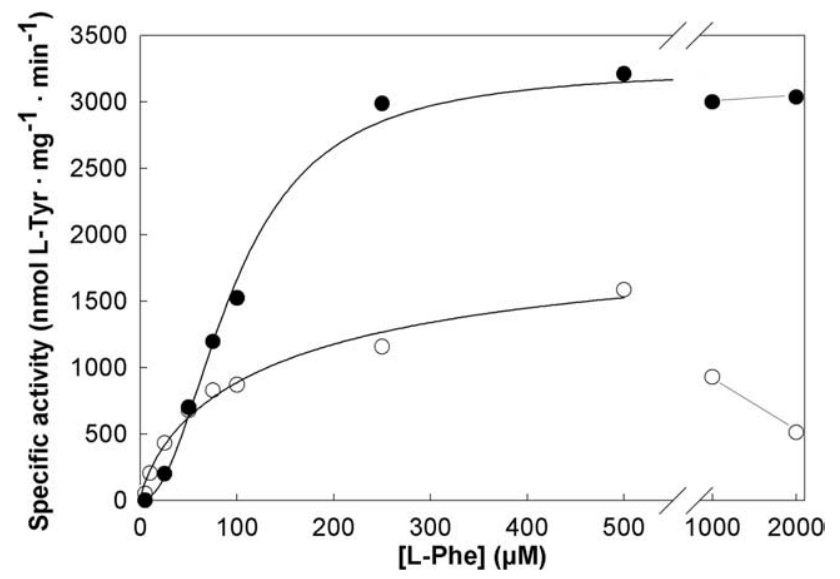

Fig. 2. Effect of L-Phe concentration on the catalytic activity of wt$\mathrm{PAH}(\bullet)$ and $\mathrm{Y} 325 \mathrm{~L}(\mathrm{O})$. The activity was measured at standard assay conditions $\left(75 \mu \mathrm{M} \mathrm{BH} 4,1\right.$ min assay, $\left.25^{\circ} \mathrm{C}\right)$ with 5 min preincubation of the enzymes at the corresponding concentrations of L-Phe at $25^{\circ} \mathrm{C}$.

\subsection{Coupling efficiency}

While oxidation of the cofactor $\mathrm{BH}_{4}$ is tightly coupled to formation of L-Tyr during normal enzyme turnover with L-Phe as substrate, the use of alternative substrates or cofactor analogues often results in uncoupling during PAH turnover, with a higher ratio of cofactor oxidation, i.e., electrons used, per substrate hydroxylation [15,23]. A partially uncoupled reaction has also been described for the two phenylketonuria associated mutations R158Q and E280K [24]. These partially uncoupled reactions are concomitantly associated to reduced specific activity measured as amino acid hydroxylation and to $\mathrm{H}_{2} \mathrm{O}_{2}$ production from the electrons not used for product formation $[15,24]$. Spectroscopic evidence revealed that the mutations R158Q and E280K also caused alterations in the iron coordination, thus rendering the protein less active [24]. As seen in Table 3, the mutants Y325L and $\mathrm{Y} 325 \mathrm{~A}$, but not $\mathrm{Y} 325 \mathrm{~F}$, also present uncoupling of the reaction $(30 \%(\mathrm{Y} 325 \mathrm{~L})$ and $80 \%(\mathrm{Y} 325 \mathrm{~A})$ of non-productive turnovers with respect to L-Tyr formation).

Table 3

Coupling efficiency of wt-PAH and the mutants at position Tyr325

\begin{tabular}{lcll}
\hline Enzyme & $\begin{array}{l}\text { Tyr produced } \\
(\mu \mathrm{mol} \mathrm{Tyr} / \\
\operatorname{min~} \mathrm{mg})\end{array}$ & $\begin{array}{l}\mathrm{BH}_{4} \text { consumed } \\
(\mu \mathrm{mol} \mathrm{NADH} / \\
\mathrm{min} \mathrm{mg})\end{array}$ & $\begin{array}{l}\text { Coupling } \\
(\mu \mathrm{mol} \mathrm{L}-\mathrm{Tyr} / \\
\mu \mathrm{mol} \mathrm{BH})\end{array}$ \\
\hline $\mathrm{Wt}$ & $3480 \pm 160$ & $3550 \pm 180$ & 0.98 \\
$\mathrm{Y} 325 \mathrm{~F}$ & $3250 \pm 400$ & $3010 \pm 350$ & 1.08 \\
$\mathrm{Y} 325 \mathrm{~L}$ & $770 \pm 50$ & $1120 \pm 230$ & 0.69 \\
Y325A & $75 \pm 5$ & $390 \pm 5$ & 0.19 \\
\hline
\end{tabular}

Measured at pH 7.0 at $25^{\circ} \mathrm{C}$ in the presence of DHPR and NADH.

\subsection{Fluorescence emission spectra}

In order to investigate if the reason why the mutant Y325L did not show positive cooperativity and activation by L-Phe was an impediment to undergo the activating L-Phe induced conformational change, we studied the conformation of the enzyme in the absence and presence of L-Phe by fluorescence spectroscopy. Human PAH contains three conserved tryptophan residues (120, 187 and 326). Activation of PAH by L-Phe has been shown to result in an increase of the intrinsic tryptophan fluoresce and a red shift of the emission maximum $[25,26]$. These changes are largely contributed by Trp120, which is supposed to exchange to a more polar environment, most probably the solvent, upon activation of the enzyme [27]. In the absence of added L-Phe, the tryptophan fluorescence emission spectrum of Y325L shows a maximum at about $337 \mathrm{~nm}$ (excitation at $295 \mathrm{~nm}$ ) and, on incubation with $1 \mathrm{mM}$ L-Phe, a 10 $\mathrm{nm}$ red-shift and an increase in the quantum yield is observed (Fig. 3). This is very similar to that observed with wt-PAH [22,27] and the silent mutant Y325F (data not shown). Thus, the activating conformational change induced by L-Phe seems to be operational in the mutant, and the lack of cooperativity observed in the activity measurements rather seems to be related to the low $S_{0.5}(\mathrm{Phe})$-value which corresponds to that of a preactivated PAH.

\subsection{EPR spectroscopy}

The ferric ion with 5 unpaired d-electrons is a high spin $5 / 2$ system. Systems with $S>1 / 2$ can be described by the standard spin Hamiltonian:

$H_{\mathrm{S}}=\beta \cdot S \cdot g \cdot B+D\left[S_{\mathbf{z}}^{2}-S(S+1) / 3+E / D\left(S_{\mathbf{x}}^{\mathbf{2}}-S_{\mathbf{y}}^{\mathbf{2}}\right)\right]$

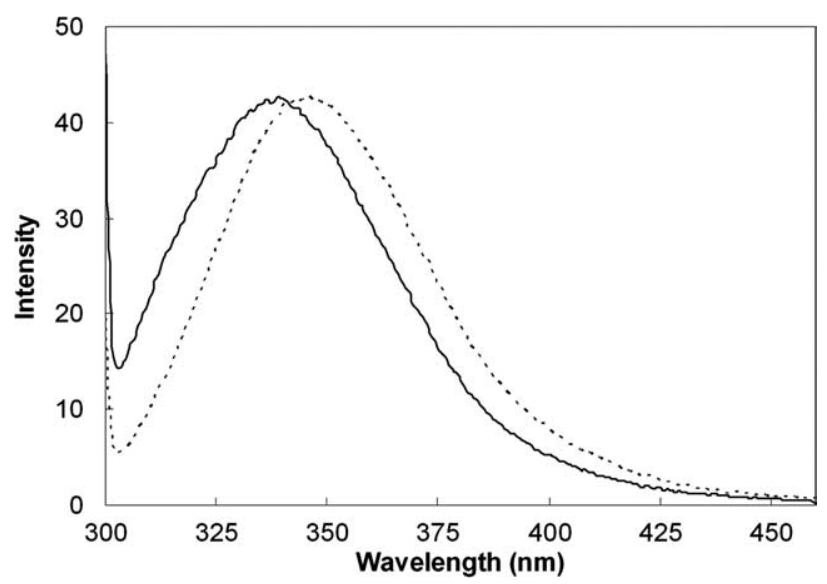

Fig. 3. Fluorescence emission spectra of the Y325L mutant of PAH The enzyme sample was diluted to a final concentration of about $1 \mu \mathrm{M}$ subunit in $20 \mathrm{mM}$ Na-HEPES, $0.2 \mathrm{M} \mathrm{NaCl}, \mathrm{pH}$ 7.0. Fluorescence spectra were acquired in the absence (-) and presence (- - ) of $1 \mathrm{mM}$ L-Phe. The excitation wavelength was $295 \mathrm{~nm}$. 
in which $\beta$ is the Bohr magneton, $B$ the applied magnetic field, $g$ the Landé tensor and $D$ and $E$ zero-field splitting parameters are the axial and the rhombic parameters, respectively. The $g, D$, and $E$ parameters in the standard spin Hamiltonian are important when discussing EPR spectra, and may give information about the metal-ligand environments [28]. The broad nature of the Fe(III) EPR spectra makes the determination of $D$ and $E$ difficult but computer simulation of the spectra provides $E / D$, which although cannot be used to predict geometry (i.e., tetrahedral versus octahedral), is a structurally useful parameter that indicates the distortion of the coordination geometry. Thus, $|E / D|$ ranges between 0 and $1 / 3$ where $|E / D|=0$ corresponds to the fully axial case and $|E / D|=1 / 3$ the fully rhombic case. As earlier described for both the rat [29-31] and human enzymes [16], the $\mathrm{X}$-band EPR spectrum of PAH shows a major signal at $g_{\text {eff }} \sim 4.3 \quad(160 \mathrm{mT})$, characteristic of rhombic $(|E / D|=0.33)$ high-spin (5/2) Fe (III) (Fig. 4(a)) from the middle Kramers doublet. These types of water-coordinated Fe(III) EPR signals are rather broad and nonresolved, and any sharp signals from the lower Kramers doublet in X-band EPR just below $g_{\text {eff }} \sim 9.7$ are hardly seen, indicating both $g$ - and $D$-strain. The spectra do not contain additional signals that could be associated to, e.g., radicals, but PAH samples usually include some contamination with small amounts of $\mathrm{Cu}(\mathrm{II})$ giving signals at the high-field region around $g=2$, and this region of the spectrum is not usually shown. Similar EPR properties were observed for the mutants Y325L and $\mathrm{Y} 325 \mathrm{~F}$, but Y325L had even less defined high spin $\mathrm{Fe}(\mathrm{III}) \mathrm{EPR}$ spectrum. At similar protein concentration Y325F presents a signal at $g_{\text {eff }} \sim 4.3$ which was similar

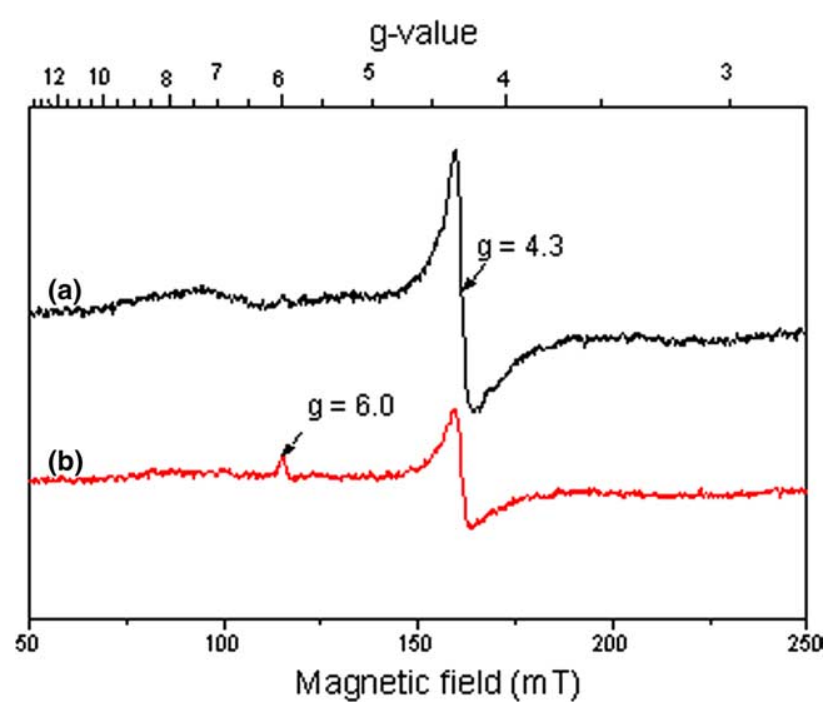

Fig. 4. EPR spectra of wt-PAH (a) and Y325L (b) as isolated. The samples $(200 \mu \mathrm{M}$ enzyme subunit) were prepared in $20 \mathrm{mM} \mathrm{Na}$ HEPES, $0.2 \mathrm{M} \mathrm{NaCl}$, pH 7.0. EPR parameters were $9.66 \mathrm{GHz}$ microwave frequency, $1 \mathrm{~mW}$ microwave power, $1 \mathrm{mT}$ modulation amplitude, and a modulation frequency of $100 \mathrm{kHz}$ at $3.6 \mathrm{~K}$. to that of wt-PAH, but Y325L shows a weaker signal, and the spectrum of this mutant also reveals an additional minor signal from a lower Kramers doublet species at $115 \mathrm{mT} g_{\perp}=6$ and $g_{\|}=2$ (Fig. 4(b)), indicating the presence of an additional small iron population with axial symmetry of the zero-field splitting $(|E / D|=0)[28]$. Integration of the EPR signal at $g=4.3$ and quantification of the iron content for the wt-PAH and the mutant forms proved that the content of iron per enzyme was nearly stoichiometric for wild-type and the Y325F mutant, while Y325L only had 0.5 atom/subunit (Table 4). Similar substoichiometric iron content for this mutant was determined by using bathophenanthrolinedisulphonic acid (Table 4). EPR spectra and iron quantifications of the wild-type and mutants taken prior and after addition of equimolar amounts of potassium ferri(III)cyanide were similar, indicating that the enzymes were isolated with the iron mainly in the ferric state. Also, while it is possible to reconstitute the ironsaturated full active wt-PAH from the iron-free apoenzyme by incubation with ferrous iron [18], the same cannot be obtained for the substoichiometrically ironsaturated mutant Y325.

Upon addition of substrate L-Phe and cofactor analogue $\mathrm{BH}_{2}$ to the enzyme samples we observed the previously reported changes induced in the EPR spectra of wt-PAH [16] also in the spectra of the mutants Y325F and $Y 325 \mathrm{~L}$, but the changes were less clear in the mutant Y325L due to the already broad signals before any ligand addition (data not shown). The simultaneous addition of both ligands leads to the disappearance of the $g_{\text {eff }}=6.0$ signal in the spectra of this mutant (data not shown). Moreover, we determined the midpoint potential $\left(E_{\mathrm{m}}(\mathrm{Fe}(\mathrm{III}) / \mathrm{Fe}(\mathrm{II}))\right)$ for the active site iron in Y325L by monitoring and measuring the intensity of the major signal at $g_{\text {eff }}=4.3$ during the redox titrations. The $\mathrm{Fe}$ (III) ion was reduced in the range of +50 to +400 $\mathrm{mV}$ and the $E_{\mathrm{m}}$-value was calculated to be $200 \pm 13 \mathrm{mV}$ (data not shown), not very different from that previously determined for wt-PAH in the absence of ligands, i.e., $+207 \pm 10 \mathrm{mV}$ [16]. The low intensity of the signal at $g_{\text {eff }}=6.0$ hindered redox titration and the calculation of the $E_{\mathrm{m}}$ for the minor iron species.

While the signal at $g=6.0$ in the EPR spectra of the mutant $\mathrm{Y} 325 \mathrm{~L}$ seems to correspond to a minor and

\section{Table 4}

Iron content for wt-PAH and mutants $\mathrm{Y} 325 \mathrm{~L}$ and $\mathrm{Y} 325 \mathrm{~F}$, determined using the chelation with bathophenanthrolinedisulphonic acid and by EPR

\begin{tabular}{lll}
\hline Enzyme & Fe/subunit $(\mu \mathrm{M} / \mu \mathrm{M})$ & EPR \\
\cline { 2 - 3 } & Bathophenanthroline & \\
\hline $\mathrm{Wt}$ & 1.04 & 1.0 \\
Y325L & 0.65 & 0.5 \\
Y325F & 1.03 & 0.8 \\
\hline
\end{tabular}


different iron species from that represented by the signal at $g=4.3$, it is not clear if this latter signal represents an iron form identical to that with the same g-value in the spectrum of wt-PAH. Indeed, the different bandwidth and shape of these signals ( $g=4.3$ region in Fig. 4(a) and (b)) indicate different iron species in the wt-PAH and the mutant. Nevertheless, the analysis of the microwave power saturation for the unligated and ligated samples did not manifest large differences between the irons in both enzyme forms (Fig. 5). Thus, at $3.6 \mathrm{~K}$,
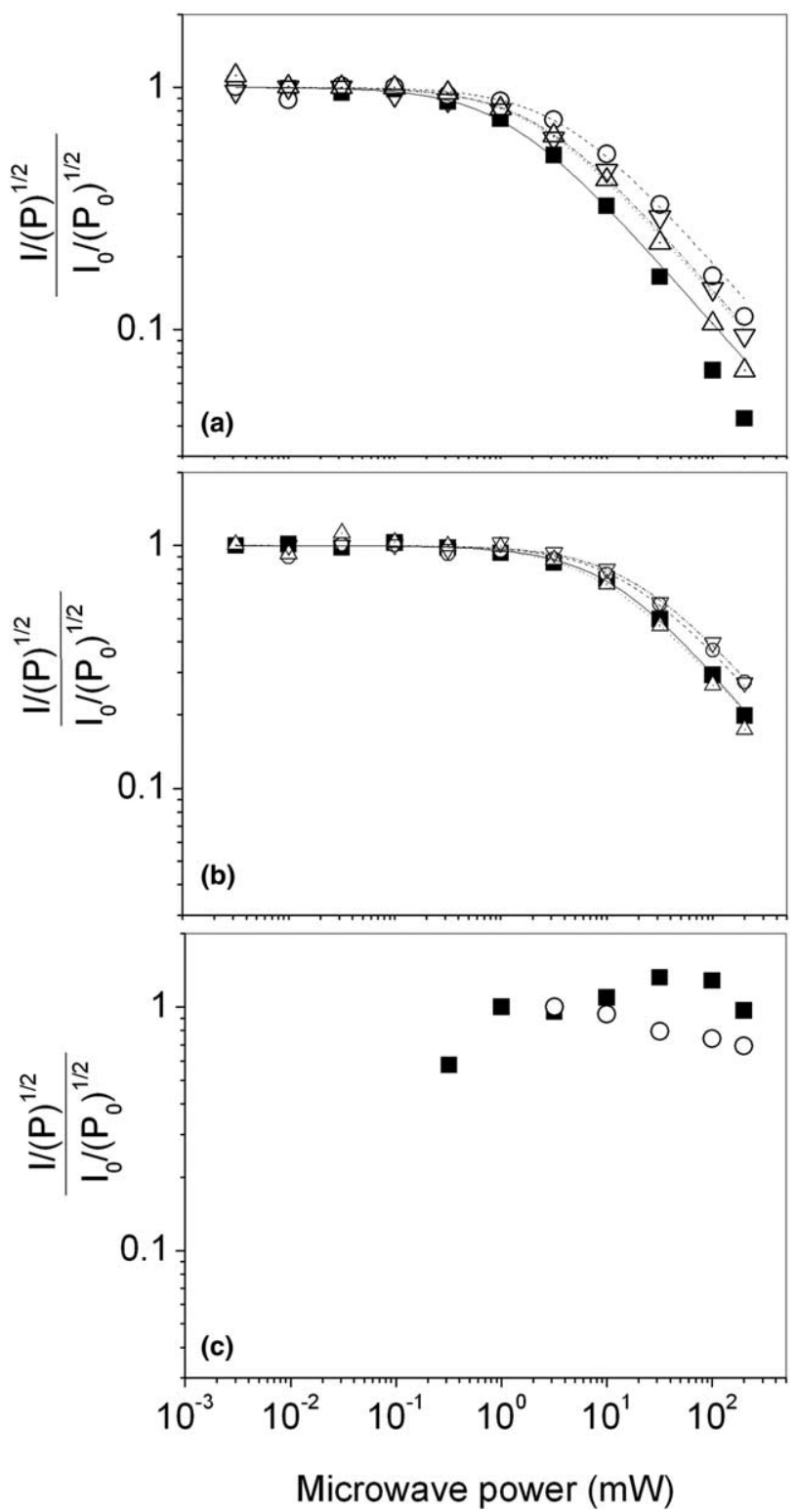

Fig. 5. Microwave power saturation analyses. Saturation behavior at two different temperatures, $3.6 \mathrm{~K}$ (a) and $10 \mathrm{~K}$ (b), of the paramagnetic species observed at $g_{\text {eff }}=4.3$ in EPR spectra of wild-type PAH in the absence ( or presence of $5 \mathrm{mM}$ L-Phe and $5 \mathrm{mM} \mathrm{BH}_{2}(\mathrm{O})$, or of mutant Y325L in the absence $(\triangle)$ or presence of $5 \mathrm{mML}_{\mathrm{L}}$-Phe and $5 \mathrm{mM} \mathrm{BH}_{2}(\nabla)$. (c) Power saturation behavior of the EPR species at $g_{\text {eff }}=6.0$ in samples of the mutant $\mathrm{Y} 325 \mathrm{~L}$, at $3.6 \mathrm{~K}(\mathbf{\square})$ or $10 \mathrm{~K}(\bigcirc)$. The curves were fitted using Eq. (1) with an inhomogeneity factor: $b \sim 1$. the half-saturation microwave power $\left(P_{1 / 2}\right)$ of the iron signal at $g=4.3$ of the wt-PAH was $1.1 \pm 0.1 \mathrm{~mW}$ for the unbound enzyme and increased to $3.6 \pm 0.4 \mathrm{~mW}$ in the presence of $\mathrm{L}-\mathrm{Phe}$ and $\mathrm{BH}_{2}$, while the unligated and ligated mutant showed $P_{1 / 2}$ between these values (Fig. 5(a)). Moreover, at $10 \mathrm{~K}$, the $P_{1 / 2}$ of the $g=4.3$ component was $8.9 \pm 1.4 \mathrm{~mW}$ for the unbound forms of both wt-PAH and $\mathrm{Y} 325 \mathrm{~L}$ and increased to $14.8 \pm 1.9$ for the ligand bound enzyme forms (Fig. 5(b)). The signals from both enzyme samples showed normal microwave power behavior with $b$ value close to 1 at both $3.6 \mathrm{~K}$ and $10 \mathrm{~K}$ (Figs. 5(a) and (b)). While these analyses indicate that the coordination environment of the major iron species is only slightly altered in the mutant with respect to the wt-PAH, the $g_{\text {eff }}=6.0$ component of the spectra of Y325L did not show normal microwave power saturation behavior, and it seems not even partially saturated at $200 \mathrm{~mW}$ (Fig. 5(c)), further demonstrating that this minor component corresponds to a different iron species. The saturation behavior of this small amount $\mathrm{Fe}$ (III) is not common, but the low intensity of the $g=6.0$ signal precludes the correct molecular interpretation of its saturation data.

\section{Discussion}

Despite the early suspected role of Tyr325 in the function of PAH [32], the conservative mutation to Phe is not appropriate to investigate the functional and structural importance of this residue due to the reversal of the mutant back to wt-PAH by posttranslational hydroxylation [9]. Accordingly, the mutant Y325F prepared in this work in full-length $\mathrm{PAH}$ showed indistinguishable properties to wt-PAH. On the other hand, the mutant Y325L is advantageous to study this location in the enzyme: opposite to mutations to Ala and Ser (Y325A and Y325S), the mutation to Leu does not have a deleterious effect on the conformational stability of the protein but has significant effects on the catalytic properties. Thus, despite its high affinity for both substrate and cofactor (Table 1) the Y325L mutant shows a reduced activity at standard conditions $(1 \mathrm{mM}$ L-Phe, $75 \mu \mathrm{M} \mathrm{BH}$ ) due to high substrate inhibition (Fig. 2) and low coupling efficiency resulting in unproductive oxidation of $\mathrm{BH}_{4}$. The lower Fe content also contributes to the reduced activity. Interestingly, despite the defective catalysis and regulation of the Y325L mutant, its catalytic efficiency measured as $V_{\max } / S_{0.5}(\mathrm{Phe})$ is comparable to that of wt-PAH. The most deleterious consequences of mutating Tyr325 are in fact the loss of iron and the uncoupling of the reaction. Loss of iron has been shown to result in a decreased conformational stability of the protein both for bacterial (C. violaceum) PAH [11] and for tyrosine hydroxylase [33]. The effects 
of the mutation are thus compatible with the important structural role of this residue by determining the close structure of the active site around the metal (Fig. 1(a)). Mutation of the homologous residue in the bacterial enzyme (Tyr179) did reveal its important role in catalysis but for that enzyme it was concluded that iron binding to the active site was not dependent on the presence of a Tyr at this position [11].

In full-length human $\mathrm{PAH}$, the substoichiometric metal binding and the altered iron coordination geometry as observed by EPR are in agreement with a mutationinduced loss of the hydrogen bond between the side chain and watl at the first coordination sphere of the catalytic iron. The signal at $g_{\text {eff }}=6.0$ observed in the EPR spectrum of the Y325L mutant is indicative of a minor iron species with axial symmetry of the zero-field splitting $(|E / D|=0)[28]$, while the $g_{\text {eff }}=4.3$ signal indicates a rhombic symmetry of the zero-field splitting $(|E / D|=0.33)$. The axial coordination geometry is often observed for heme $\mathrm{Fe}$ (III) and is less common for nonheme $\mathrm{Fe}(\mathrm{III})$ proteins, but it has been observed for, e.g., lipoxygenase [34] and in catechol complexes of PAH and tyrosine hydroxylase $[35,36]$. In PAH the enzymatic active iron can be converted to axial species under different conditions $[16,29,30]$.

The rhombic geometry observed for the iron in the resting unligated ferric state of wt-PAH by EPR is in accordance with the six-coordinate distorted octahedral site revealed by both crystallography (Fig. 1(a)) and Xray absorption spectroscopy for the ferrous form [4,37]. The ferrous active site adopts a five-coordinate square pyramidal structure upon substrate analogue and cofactor binding, which results in the bidentate coordination of the glutamate ligand and the elimination of two water ligands $[37,38]$. Release of a soft ligand, usually water, upon substrate binding is a common feature in several non-heme iron proteins containing the 2-His-1-carboxylate facial triad motif $[39,40]$. Releasing of the water ligand occurs trans to any of the three residues in the coordinating motif, allowing the binding of $\mathrm{O}_{2}$ [39]. Wat1, which is hydrogen bonded $(2.8 \AA)$ to $\operatorname{Tyr} 325$, is trans to His 285 and is released upon cofactor and substrate binding (Fig. 1(b)). The substitution of this residue by a leucine would disrupt the hydrogen-bonding network between the iron, wat1, and the phenolate oxygen in Tyr325 and would result in both the delocalization and/or partial release of this water ligand and the reorganization of the first coordination sphere of the ferric iron, in accordance with (i) the increased axial geometry of part of the iron environment, (ii) the lower affinity for the iron in the mutant, and (iii) the higher $P_{1 / 2}$ values of the signal at $g=6.0$, and to less degree at $g=4.3$, indicative of a higher heterogeneity of the iron coordination environment. Moreover, the low $S_{0.5}(\mathrm{Phe})$ and $K_{\mathrm{m}}\left(\mathrm{BH}_{4}\right)$ values in Y325L (Table 1) are also in agreement with the binding of the ligands being facilitated when wat1 binds less tighter or is absent, since this water would behave as a competitive ligand to substrate and cofactor binding in wt-PAH. Mutation of Tyr325 in the rat enzyme [7], or of the corresponding residue in C. violaceum [11] and tyrosine hydroxylase (Tyr371) [8], also are accompanied by a higher affinity for L-Phe. This higher binding affinity for the substrates does not seem to be paralleled by their correct binding in $\mathrm{Y} 325 \mathrm{~L}$, as indicated by the partial uncoupling of the reaction compatible with a defective formation of the quaternary $\mathrm{PAH} \cdot \mathrm{Fe}(\mathrm{II}) \cdot \mathrm{BH}_{4} \cdot \mathrm{Phe} \cdot \mathrm{O}_{2}$ complex. In addition, Tyr325 appears to be important for the correct cooperative regulation of PAH by L-Phe, which is crucial to maintain L-Phe homeostasis in vivo [1].

In summary, although the significant residual activity of the mutant Y325L does not support the early hypothesis that Tyr325 has an essential mechanistic role, by, e.g., stabilizing a radical intermediate [4], our results clearly prove the important role of this residue in human $\mathrm{PAH}$ in determining the iron binding stoichiometry, the architecture of the iron site and the right positioning of the substrate and cofactor.

\section{Abbreviations}

\begin{tabular}{|c|c|}
\hline $\mathrm{BH}_{2}$ & L-erythro-7,8-dihydrobiopterin \\
\hline $\mathrm{BH}_{4}$ & $(6 R)$-L-erythro-5,6,7,8-tetrahydrobiopterin \\
\hline DHPR & dihydropteridin reductase \\
\hline$E_{\mathrm{m}}$ & midpoint oxidation-reduction potential \\
\hline EPR & electron paramagnetic resonance \\
\hline HEPES & $\begin{array}{l}N \text {-2-hydroxyethylpiperazine- } N^{\prime} \text { - } \\
\text { ethanesulphonic acid }\end{array}$ \\
\hline $\mathrm{PAH}$ & phenylalanine hydroxylase \\
\hline PCR & polymerase chain reaction \\
\hline$P_{1 / 2}$ & $\begin{array}{l}\text { microwave power at } 50 \% \text { saturation of the } \\
\text { EPR signal }\end{array}$ \\
\hline $\mathrm{w}$ & wild-type \\
\hline
\end{tabular}

\section{Acknowledgments}

We thank Randi M. Svebak and Øyvind Halskau for expert technical assistance and valuable help. This work was supported by The Research Council of Norway and the Fundação para a Ciência e a Tecnologia, Portugal.

\section{References}

[1] C.R. Scriver, S. Kaufman, Hyperphenylalaninemia: phenylalanine hydroxylase defciency, in: C.R. Scriver, A.L. Beaudet, D. Valle, W.S. Sly (Eds.), The Metabolic and Molecular Bases of Inherited Disease, McGraw-Hill, New York, 2001, pp. 1667-1724.

[2] P.F. Fitzpatrick, Biochemistry 42 (2003) 14083-14091.

[3] S. Kaufman, Adv. Enzymol. Relat. Areas Mol. Biol. 67 (1993) $77-$ 264. 
[4] H. Erlandsen, F. Fusetti, A. Martínez, E. Hough, T. Flatmark, R.C. Stevens, Nat. Struct. Biol. 4 (1997) 995-1000.

[5] O.A. Andersen, T. Flatmark, E. Hough, J. Mol. Biol. 314 (2001) 266-278.

[6] H. Erlandsen, E. Bjørgo, T. Flatmark, R.C. Stevens, Biochemistry 39 (2000) 2208-2217.

[7] I.G. Jennings, R.G. Cotton, B. Kobe, Arch. Biochem. Biophys. 384 (2000) 238-244.

[8] S.C. Daubner, P.F. Fitzpatrick, Biochemistry 37 (1998) 1644016444.

[9] S.D. Kinzie, M. Thevis, K. Ngo, J. Whitelegge, J.A. Loo, M.M. Abu-Omar, J. Am. Chem. Soc. 125 (2003) 4710-4711.

[10] M.J. Ryle, R.P. Hausinger, Curr. Opin. Chem. Biol. 6 (2002) 193 201.

[11] J. Zoidakis, M. Sam, A. Volner, A. Han, K. Vu, M.M. AbuOmar, J. Biol. Inorg. Chem. 9 (2004) 289-296.

[12] H.G. Eiken, P.M. Knappskog, J. Apold, T. Flatmark, Hum. Mutat. 7 (1996) 228-238.

[13] A. Martínez, P.M. Knappskog, S. Olafsdottir, A.P. Døskeland, H.G. Eiken, R.M. Svebak, M. Bozzini, J. Apold, T. Flatmark, Biochem. J. 306 (1995) 589-597.

[14] F.F. Miranda, K. Teigen, M. Thorolfsson, R.M. Svebak, P.M. Knappskog, T. Flatmark, A. Martínez, J. Biol. Chem. 277 (2002) 40937-40943.

[15] M.D. Davis, S. Kaufman, Neurochem. Res. 16 (1991) 813-819.

[16] P.L. Hagedoorn, P.P. Schmidt, K.K. Andersson, W.R. Hagen, T. Flatmark, A. Martinez, J. Biol. Chem. 276 (2001) 2285022856

[17] K.R. Strand, S. Karlsen, K.K. Andersson, J. Biol. Chem. 277 (2002) 34229-34238.

[18] S. Olafsdottir, A. Martínez, J. Biol. Chem. 274 (1999) 6280-6284.

[19] D. Blair, H. Diehl, Talanta 7 (1961) 163-174.

[20] R. Aasa, T. Vanngard, J. Magn. Reson. 19 (1975) 308-315.

[21] J.A. Fee, Methods Enzymol. 49 (1978) 512-528.

[22] M. Thórólfsson, K. Teigen, A. Martínez, Biochemistry 42 (2003) 3419-3428.
[23] T.J. Kappock, J.P. Caradonna, Chem. Rev. 96 (1996) 2659 2756.

[24] J.N. Kemsley, E.C. Wasinger, S. Datta, N. Mitic, T. Acharya, B. Hedman, J.P. Caradonna, K.O. Hodgson, E.I. Solomon, J. Am. Chem. Soc. 125 (2003) 5677-5686.

[25] J.J. Marota, R. Shiman, Biochemistry 23 (1984) 1303-1311.

[26] R.S. Phillips, M.A. Parniak, S. Kaufman, Biochemistry 23 (1984) 3836-3842.

[27] P.M. Knappskog, J. Haavik, Biochemistry 34 (1995) 1179011799.

[28] R. Cammack, C.E. Cooper, Methods Enzymol. 227 (1993) 353 384.

[29] D.E. Wallick, L.M. Bloom, B.J. Gaffney, S.J. Benkovic, Biochemistry 23 (1984) 1295-1302.

[30] A. Martínez, K.K. Andersson, J. Haavik, T. Flatmark, Eur. J. Biochem. 198 (1991) 675-682.

[31] T.J. Kappock, P.C. Harkins, S. Friedenberg, J.P. Caradonna, J. Biol. Chem. 270 (1995) 30532-30544.

[32] H. Erlandsen, A. Martinez, P.M. Knappskog, J. Haavik, E. Hough, T. Flatmark, FEBS Lett. 406 (1997) 171-174.

[33] A. Martínez, J. Haavik, T. Flatmark, J.L.R. Arrondo, A. Muga, J. Biol. Chem. 271 (1996) 19737-19742.

[34] S. Slappendel, G.A. Veldink, J.F. Vliegenthart, R. Aasa, B.G. Malmstrom, Biochim. Biophys. Acta 667 (1981) 77-86.

[35] D.D. Cox, S.J. Benkovic, L.M. Bloom, F.C. Bradley, M.J. Nelson, L. Que, D.E. Wallick, J. Am. Chem. Soc. 110 (1988) 2026-2032.

[36] K.K. Andersson, D.D. Cox, L. Que Jr., T. Flatmark, J. Haavik, J. Biol. Chem. 263 (1988) 18621-18626.

[37] E.C. Wasinger, N. Mitic, B. Hedman, J. Caradonna, E.I. Solomon, K.O. Hodgson, Biochemistry 41 (2002) 6211-6217.

[38] O.A. Andersen, T. Flatmark, E. Hough, J. Mol. Biol. 320 (2002) 1095-1108.

[39] L. Que Jr., Nat. Struct. Biol. 7 (2000) 182-184.

[40] E.I. Solomon, A. Decker, N. Lehnert, Proc. Natl. Acad. Sci. USA 100 (2003) 3589-3594. 